\title{
Estudio epidemiológico de los casos de triquinelosis registrados en la provincia de Santa Fe, Argentina, 1998-2009
}

\author{
Gabriel J. Sequeira, ${ }^{1}$ María L. Dalla Fontana, ${ }^{2}$ María V. Zbrun, ${ }^{3}$ \\ Lorena P. Soto, ${ }^{4}$ Laureano S. Frizzo, ${ }^{4}$ María del Pilar Zarazaga, ${ }^{1}$ \\ Inés C. Sanchez ${ }^{1}$ y Marcelo L. Signorini ${ }^{4}$
}

Forma de citar Sequeira GJ, Dalla Fontana ML, Zbrun MV, Soto LP, Frizzo LS, Zarazaga MP, et al. Estudio epidemiológico de los casos de triquinelosis registrados en la provincia de Santa Fe, Argentina, 1998-2009. Rev Panam Salud Publica. 2013;33(5):363-9.

RESUMEN Objetivo. Caracterizar desde los puntos de vista epidemiológico, clínico y de laboratorio los casos y brotes de triquinelosis que se registraron en la provincia de Santa Fe, Argentina, entre 1998 y 2009.

Métodos. Se analizaron 1519 fichas epidemiológicas de personas que presentaron síntomas y signos compatibles con triquinelosis en la provincia de Santa Fe en el período 1998-2009. Se realizó el análisis estadístico descriptivo de la información contenida en las fichas epidemiológicas y los resultados clínicos y de laboratorio; se aplicó el análisis bifactorial para determinar las posibles asociaciones con factores epidemiológicos y ambientales.

Resultados. Se encontraron sintomas de triquinelosis en 1276 casos; 372 cumplian la definición de caso clínico y 224 muestras resultaron positivas por inmunofluorescencia indirecta, independientemente de su clasificación como caso clinico. Se identificaron 27 brotes que involucraron a 1157 casos en ocho departamentos, con mayor frecuencia en el centro y el sur de la provincia. La distribución anual de los casos asociados epidemiológicamente entre sí fue heterogénea, con una mayor frecuencia en el período 2000-2003 y en los meses de agosto a octubre. Hubo mayor probabilidad de enfermar cuando se consumieron productos de fuentes informales $(O R=3,69 ; \mathrm{P}=0,014)$ y en las áreas rurales $(O R=1,799 ; \mathrm{P}=0,011)$. El período de incubación de la enfermedad (mediana) fue de 12 dias. Las personas que consumieron carne de cerdo o sus derivados tuvieron 2,06 veces más riesgo de enfermar que las que no la consumieron. Conclusiones. El mayor número de brotes ocurrió en los departamentos más poblados y con mayor producción porcina. Se deben diseñar acciones específicas de prevención y control de la enfermedad en esos departamentos. Se debe considerar el análisis epidemiológico de riesgos al evaluar, gestionar y comunicar los riesgos sanitarios de triquinelosis.

Palabras clave Triquinelosis; porcinos; epidemiología; Argentina.

1 Facultad de Ciencias Agropecuarias, Universidad Católica de Córdoba, Córdoba, Argentina. La correspondencia se debe dirigir a Gabriel J. Sequeira. Correo electrónico: sequeiragabriel@gmail.com

2 Laboratorio de Zoonosis, Ministerio de Salud de la Provincia de Santa Fe, Santa Fe, Argentina.

3 Departamento Salud Pública, Facultad de Ciencias Veterinarias, Universidad Nacional del Litoral, Santa Fe, Argentina.

4 Consejo Nacional de Ciencia y Técnica, Instituto Nacional de Tecnología Agropecuaria, Santa Fe, Argentina.
La triquinelosis es una zoonosis parasitaria transmitida por alimentos, causada por nematodos intestinales del género Trichinella. Aunque las especies de este género tienen una distribución mundial y pueden infestar a mamíferos, aves y reptiles, en Argentina solo se ha documentado la presencia de la especie $T$. spiralis, que afecta a cerdos $y$ mamíferos silvestres y se transmite al hombre cuando consume esas carnes sin el tratamiento adecuado. Esta parasitosis constituye un grave problema de salud pública, dadas la alta tasa de morbilidad y las pérdidas económicas que ocasiona, especialmente en zonas endémicas. Su incidencia en humanos, no obstante, es variable y depende en parte de las prác- 
ticas relacionadas con la ingestión y la preparación de las carnes de las especies hospederas $(1,2)$.

El ciclo biológico de las especies de Trichinella tiene una fase silvestre, en la cual la triquinelosis se transmite y perpetúa en animales salvajes, y una fase doméstica o peridoméstica, en la que se transmite y perpetúa en animales domésticos, fundamentalmente cerdos y especies sinantrópicas — como los roedores-, además de gatos, perros, zorros y armadillos (1). En esta última fase, el parásito se transmite entre los cerdos, especialmente por la ingestión de desechos que contienen fibras musculares de origen porcino o canibalismo. Por ese motivo, la incidencia en los cerdos es particularmente alta cuando se los alimenta con residuos de cocinas, restaurantes y mataderos, o cuando se los mantiene en basurales de las poblaciones urbanas cercanas. Las larvas enquistadas son muy resistentes a la putrefacción, de modo que otra fuente de infección puede ser la carne de animales muertos, entre ellos las ratas, que con frecuencia se encuentran muertas en los basurales (3).

La enfermedad tiene un período de incubación promedio de 10 días y las manifestaciones clínicas varían grandemente en dependencia de las tres fases de la enfermedad: intestinal, muscular y convalecencia (3-5). Aunque el diagnóstico clínico se puede dificultar por esa diversidad de signos y síntomas clínicos, se considera que la presencia concomitante de mialgia, edema bipalpebral y eosinofilia indica una posible triquinelosis y llevaría a recomendar hacer pruebas de laboratorio para confirmar la enfermedad. No obstante, la ausencia de la conjunción de estos tres signos y síntomas no es un criterio suficiente para descartar la enfermedad.

Debido a la ausencia de síntomas específicos durante la etapa intestinal, el diagnóstico clínico solo se puede realizar a partir de la fase muscular de la enfermedad. El diagnóstico confirmatorio de laboratorio se basa fundamentalmente en métodos serológicos — como análisis inmunoenzimático por ELISA, inmunofluorescencia indirecta (IFI) e inmunotransferencia enzimática (Western blot)_ que se basan en la detección de anticuerpos de la clase IgG, que aparecen entre 12 y 60 días después de la infestación inicial. El tiempo de seroconversión depende de varios factores, como el número de larvas ingeridas, las especies de Trichinella implicadas y la respuesta inmunitaria individual (4). También se han empleado técnicas moleculares para el diagnóstico de la triquinelosis (6).

En Argentina se registraron 8806 casos de esta enfermedad en humanos entre 1990 y 2006 (7), con registros en 18 de las 23 provincias del país (1). En Santa $\mathrm{Fe}$, una de las principales productoras de ganado porcino del país, se han presentado casos o brotes en la última década, por lo que se la considera un área endémica de la enfermedad (8).

En este contexto, el análisis de riesgos es un instrumento que permite evaluar los posibles vínculos entre los problemas presentes en la cadena alimentaria y los riesgos para la salud humana, a partir de la evaluación, la gestión y la comunicación de la información (9).

El objetivo del presente trabajo fue caracterizar desde los puntos de vista epidemiológico, clínico y de laboratorio los casos y brotes de triquinelosis que se registraron en la provincia de Santa $\mathrm{Fe}$, Argentina, entre 1998 y 2009.

\section{MATERIALES Y MÉTODOS}

Se analizaron 1519 fichas epidemiológicas de personas que presentaron síntomas y signos compatibles con triquinelosis en la provincia de Santa Fe en el período 1998-2009. Los servicios de salud de la provincia enviaron al Laboratorio de Zoonosis, dependiente del Ministerio de Salud de la Provincia de Santa Fe, las fichas epidemiológicas con muestras de sangre para el análisis serológico de los casos clínicos o los brotes de triquinelosis, cuando se sospechó la enfermedad.

A los efectos del análisis de la información de los casos registrados se utilizaron las siguientes definiciones específicas:

- Caso clínico: paciente que presentó eosinofilia, mialgia y edema bipalpebral

- Caso epidemiológico: paciente que respondía a la definición de caso clínico y además estaba relacionado epidemiológicamente con otros casos clínicos o con un brote

- Caso confirmado: paciente que respondía a la definición de caso clínico con diagnóstico confirmado por IFI

- Brote: dos o más casos clínicos asociados entre sí por la fecha del comienzo de los síntomas y la localidad, con resultado positivo de laboratorio por lo menos en un caso.
La confirmación de laboratorio se basó en la detección de la seroconversión, es decir, cuando se comprobó que el título de anticuerpos aumentó en al menos dos muestras serológicas pareadas con un intervalo de 15 días entre la primera y la segunda, e intervalos de 20 días entre las subsiguientes muestras a partir de la segunda, según se establece en el protocolo de actuación ante casos sospechados de triquinelosis del Laboratorio de Zoonosis de la provincia de Santa Fe. Cuando no se contaba con dos muestras pareadas se consideraron confirmados los casos con asociación epidemiológica y título de anticuerpos mayor de 1/64.

Se realizó el análisis estadístico descriptivo de la información contenida en las fichas epidemiológicas y se aplicó el análisis bifactorial mediante la prueba de la ji al cuadrado para determinar la posible asociación de las variables independientes de los pacientes (edad, sexo, lugar de trabajo, área de residencia y consumo de posibles alimentos portadores) con las variables de resultado (caso clínico y caso sospechado). Se estimó el riesgo mediante la razón de posibilidades (odds ratio, OR) con los programas estadísticos Epi Info 2002 (Centros para el Control y Prevención de Enfermedades, Atlanta, Estados Unidos de América) e InfoStat (Universidad Nacional de Córdoba, Argentina). Se utilizó un nivel de significación de $95 \%$.

\section{RESULTADOS}

De los 1519 casos registrados, se encontraron síntomas o signos de triquinelosis en $1276 \mathrm{y}$, de ellos, en 372 el conjunto de síntomas y signos cumplían la definición de caso clínico. Por otra parte, solamente 224 muestras del total resultaron positivas según la prueba de IFI.

\section{Elementos epidemiológicos}

Se identificaron 27 brotes que involucraron a 1157 casos asociados epidemiológicamente entre sí en 8 de los 19 departamentos de la provincia, con una mayor frecuencia de casos en los departamentos del centro y el sur (figura 1).

En general, 41,3\% de los 1519 casos registrados eran mujeres y 58,7\% eran varones; las edades fluctuaron entre $1 \mathrm{y}$ 85 años, aunque las mayores frecuencias se observaron en personas de 11 a 50 años (figura 2). No se encontró asociación estadísticamente significativa entre 
FIGURA 1. Distribución de los brotes y casos clínicos de triquinelosis por departamentos, Provincia de Santa Fe, Argentina, 1998-2009

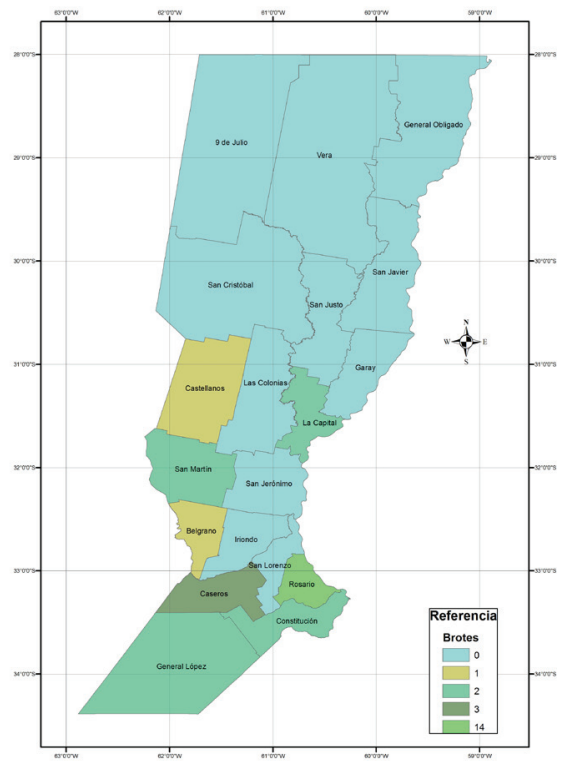

Brotes de triquinelosis

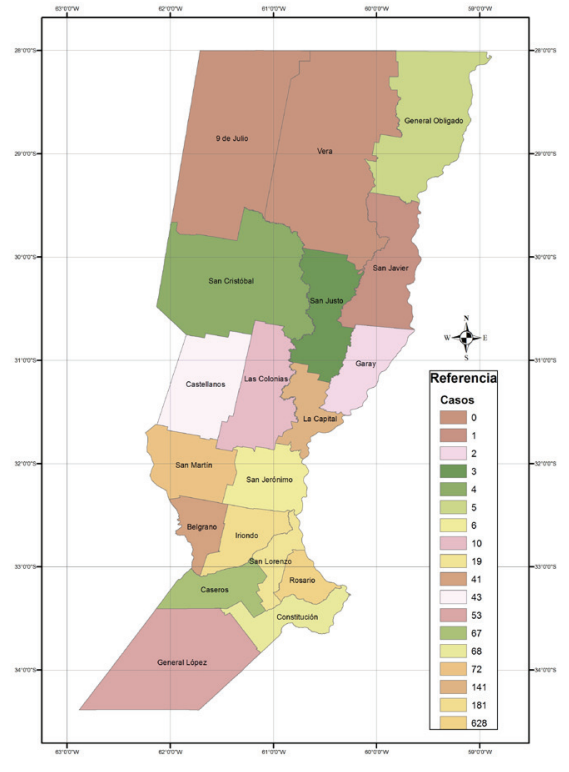

Casos de triquinelosis
FIGURA 2. Frecuencia de casos clínicos de triquinelosis, según el grupo de edad y el sexo, Santa Fe, Argentina, 1998-2009

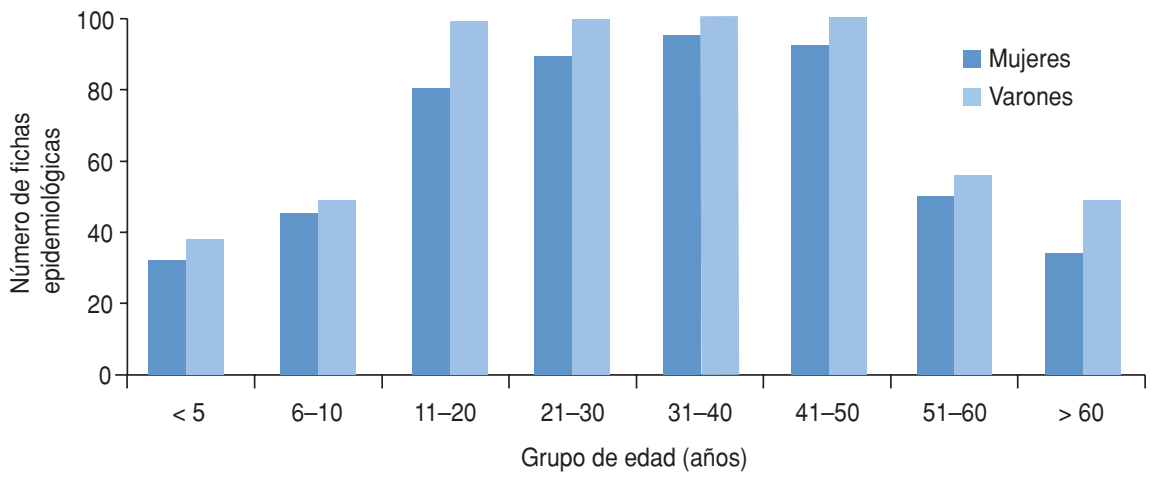

el sexo y la ocupación de las personas con los casos confirmados $(P=0,729$ y $P=0,873$, respectivamente), posiblemente debido a la gran variedad de profesiones representadas.

La distribución por año de los casos clínicos fue heterogénea a lo largo de la serie analizada (1998-2009). Después del primer bienio (1998-1999) en el que se registró $12,9 \%$ del total de los casos clínicos del período, se llegó a un pico de 40,2\% (bienio 2000-2001) y volvió a bajar sostenidamente la frecuencia relativa en los siguientes bienios (2002-2003: 24,6\%; 2004-2005: 8,5\%; 2006-2007: 2,8\%). No obstante, la frecuencia de casos volvió a aumentar en el último bienio analizado (2008-2009) hasta representar el 10,8\% del total de los casos clínicos registrados en el período de estudio.

Cuando se analizó la distribución mensual de los casos clínicos (tomando como referencia la fecha de inicio de los síntomas), se observó una mayor frecuencia en los meses de agosto y septiembre, seguidos más de lejos por octubre; en esos tres meses se concentró el 55,0\% de esos casos (figura 3).

El mayor número de casos clínicos se registró en personas que residían en áreas urbanas, pero al asociar esa información con los casos confirmados se en- contró una mayor probabilidad de enfermar en las personas que vivían en áreas rurales con respecto a las que vivían en las ciudades (OR $=1,799 ; P=0,011)$.

Los alimentos asociados epidemiológicamente con los casos clínicos fueron muy variados, pero todos contenían o estaban elaborados con carne de cerdo, o embutidos, salamines y chorizos frescos. No hubo asociación entre el tipo de producto que consumieron las personas y los casos confirmados $(P=0,839)$.

Cuando se analizó la fuente de los alimentos, ya fuera formal (con controles sanitarios o de origen comercial) o informal (sin controles sanitarios, de comercialización clandestina o elaborados para el consumo familiar), se encontró una mayor probabilidad de enfermar cuando se consumieron productos procedentes de fuentes informales que de formales $(\mathrm{OR}=3,69 ; P=0,014)$.

\section{Aspectos clínicos}

El período de incubación de la enfermedad -desde el momento de la ingestión del alimento contaminado hasta la aparición de los primeros síntomasvarió entre 3 y 80 días, con una mediana de 12: con 6 días en el primer cuartil y 20 días en el cuarto.

Los síntomas más frecuentes fueron los relacionados con la definición de caso clínico, es decir, mialgia, edema bipalpebral y eosinofilia. La mialgia fue el síntoma clínico más frecuente en todos los grupos de edad, excepto en los menores de 5 años, en los que el síntoma más frecuente fue la fiebre; el edema bipalpebral y la eosinofilia tuvieron frecuencias similares en todos los grupos de edad (figura 4).

Solo en 109 (29,3\%) de los casos clínicos se confirmó la enfermedad con las pruebas de laboratorio, mientras que 115 $(16,2 \%)$ de las personas que no presentaron la totalidad de los síntomas que definían a los casos clínicos resultaron positivas al análisis de laboratorio.

La relación entre los casos clínicos y los casos confirmados fue significativa (OR $=2,14 ; P<0,001)$. Sin embargo, las personas que no presentaban al menos uno de los tres síntomas o signos característicos de la enfermedad presentaron una probabilidad de $83,8 \%$ de resultar negativos por IFI (valor predictivo negativo de la prueba), mientras que las personas que presentaban los tres síntomas tuvieron una probabilidad de $29,3 \%$ 
FIGURA 3. Distribución mensual de los casos clínicos de triquinelosis asociados epidemiológicamente entre sí, Santa Fe, Argentina, 1998-2009

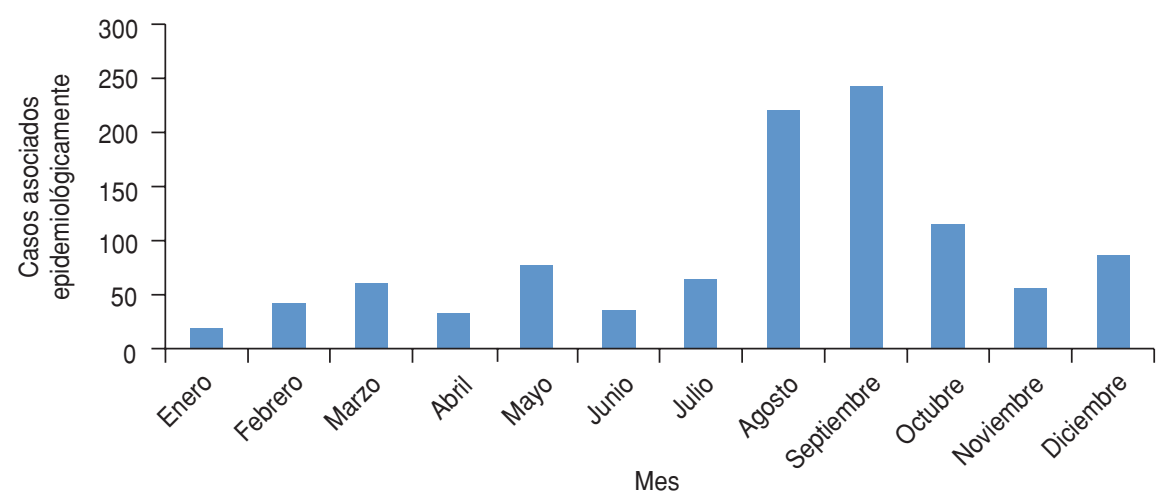

FIGURA 4. Frecuencia de los signos y los síntomas en los casos clínicos de triquinelosis, por grupo de edad, Santa Fe, Argentina, 1998-2009

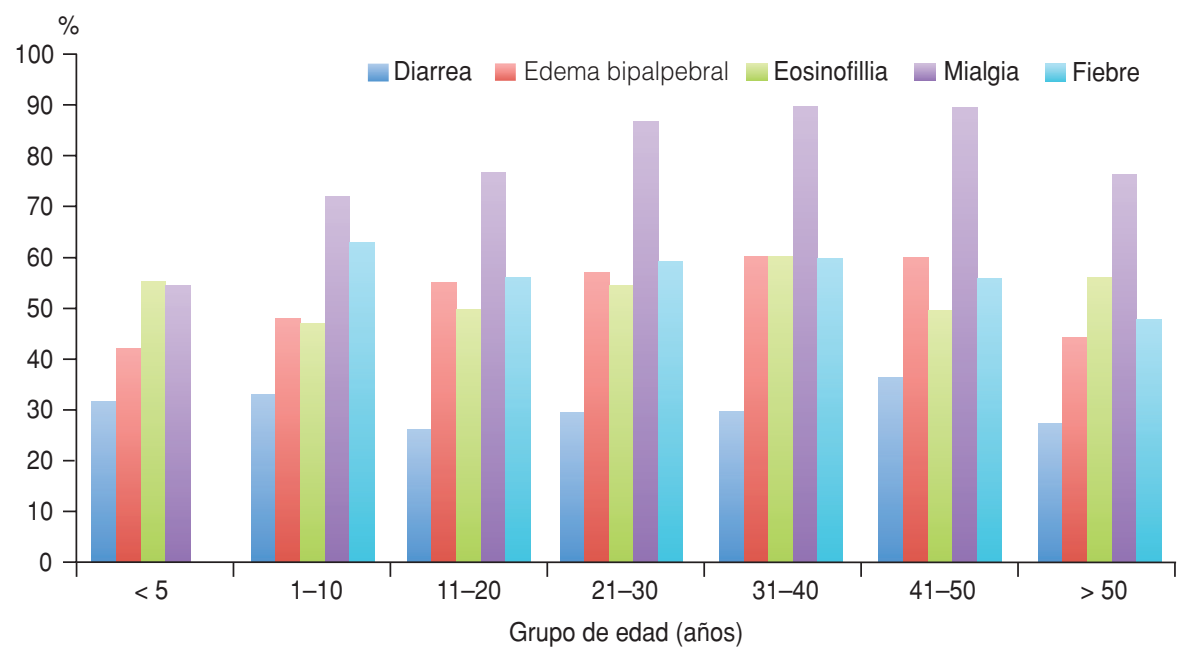

CUADRO 1. Relación entre los casos clínicos ${ }^{a}$ y los resultados de la prueba de inmunofluorescencia indirecta (IFI), Santa Fe, Argentina, 1998-2009

\begin{tabular}{lccr}
\hline & \multicolumn{2}{c}{ Resultados de la prueba de IFI } & \\
\cline { 2 - 3 } \multicolumn{1}{r}{ Caso clínico } & Positivos (\%) & Negativos (\%) & Total \\
\hline Sí & $109(29,3)$ & $263(70,7)$ & 372 \\
No & $115(16,2)$ & $594(83,8)$ & 709 \\
Total & $224(20,7)$ & $857(79,3)$ & 1081 \\
\hline
\end{tabular}

a Se consideraron casos clínicos los que presentaron edema bipalpebral, mialgia y eosinofilia mayor de $4 \%$.

de resultar positivos (valor predictivo positivo de la prueba) (cuadro 1).

$\mathrm{Al}$ analizar los resultados confirmatorios de las pruebas de laboratorio con los antecedentes epidemiológicos de haber comido carne de cerdo o sus derivados, se observó una asociación significativa $(\mathrm{OR}=2,06 ; P<0,001)$, es decir, las per- sonas que habían consumido carne de cerdo (o sus derivados) tenían 2,06 veces más riesgo de enfermar que las que no la habían consumido.

Al analizar los casos clínicos —que presentaban todos los síntomas y signos característicos de la enfermedad- que además tenían antecedentes epidemioló- gicos - haber consumido carne de cerdo o sus derivados-, el porcentaje de casos confirmados aumentó a 59,4\%.

\section{Resultados de laboratorio}

De los casos registrados, $44,8 \%$ llegaron o sobrepasaron el valor de $4 \%$ de eosinofilia, criterio distintivo de la triquinelosis clínica. Sin embargo, 74,1\% de esos pacientes resultaron negativos por IFI (falsos positivos) y $14,5 \%$ de los que no presentaron eosinofilia fueron positivos a esa prueba (falsos negativos). Estos resultados son importantes y se deben tener en cuenta para evitar que la presencia de este signo se utilice como confirmación de la enfermedad, sin otras consideraciones adicionales.

El diagnóstico serológico por IFI presentó una gran variabilidad en las cuatro muestras de un mismo paciente analizadas, especialmente entre la tercera y la cuarta muestras (figura 5). Si bien en 291 casos clínicos se obtuvo el resultado positivo a la prueba de IFI en la primera muestra y en 426 casos clínicos en la segunda, solamente resultaron positivos 148 en la tercera muestra y 63 en la cuarta. A partir del total de muestras con título de anticuerpos por IFI $(n=568)$, solo en $155(27,3 \%)$ casos se pudo comprobar la seroconversión.

De las 225 muestras de casos asociados con los tres brotes epidemiológicos a las que se le realizaron pruebas de IFI y ELISA, solo 56 resultaron positivas por ambas técnicas y 31 resultaron positivas por ELISA y negativas por IFI; en estos casos no se analizó la seroconversión.

\section{DISCUSIÓN}

En general, 76,8\% de los casos clínicos estudiados se registraron en ocho departamentos de la provincia de Santa $\mathrm{Fe}$, en los que se concentra $76,8 \%$ de la producción primaria de ganado porcino de la provincia (10).

La distribución anual de casos clínicos varió considerablemente en los bienios del período analizado. Independientemente del incremento observado en el último bimestre, el número de casos clínicos de triquinelosis informados se ha reducido. Esto concuerda con los datos del Sistema de Vigilancia Epidemiológica del Ministerio de Salud de Argentina, que muestran que el número de casos de triquinelosis ha disminuido en todo el país, al igual que el número de 
FIGURA 5. Resultados de la prueba de inmunofluorescencia indirecta (IFI) en las muestras seriadas tomadas de los casos clínicos de triquinelosis, Santa Fe, Argentina, 1998-2009

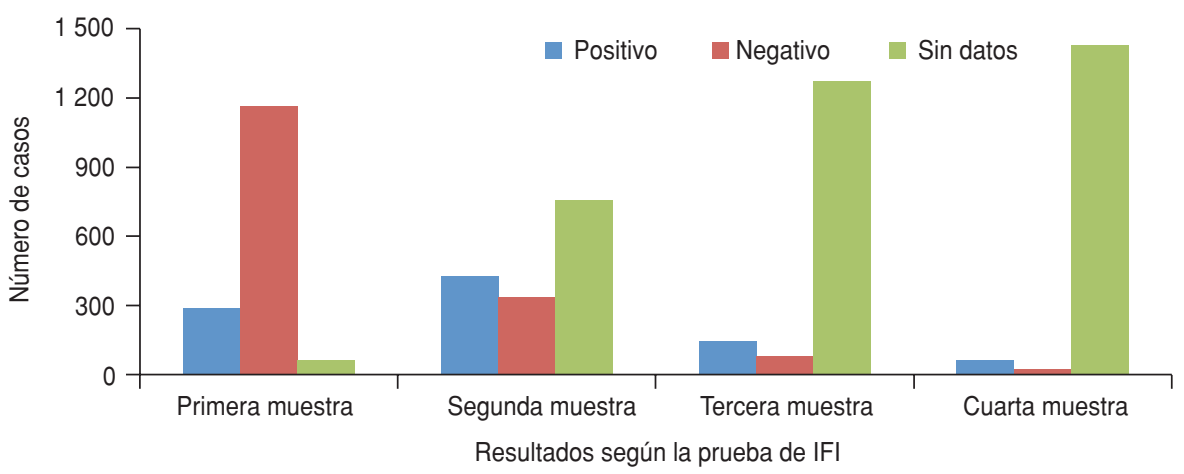

brotes (11). En la provincia de Santa Fe, en el bienio 2007-2009 solo se informó un brote de la enfermedad, mientras que en el período 2000-2003 se concentró el mayor número de brotes y de casos clínicos registrados. En Argentina se viene trabajando intensamente en campañas para la erradicación de los criaderos informales de cerdos (en su mayoría alimentados con residuos domésticos) y en elevar el nivel de conciencia de la población para que eviten el consumo de productos porcinos que no posean la etiqueta del elaborador con el sello de la autoridad sanitaria encargada de certificar la inocuidad de dichos productos. No obstante, aún se siguen presentando brotes de la enfermedad, concentrados en sectores socialmente vulnerables, donde aún se crían cerdos en traspatios, alimentados a partir de los basurales de las grandes ciudades.

El aumento en el riesgo de adquirir la enfermedad a medida que aumenta la edad puede deberse a una mayor ingestión de carne y derivados de cerdo (incremento de la exposición) o a una mayor tasa de notificación de casos, aunque estas hipótesis deben ser investigadas con mayor detalle, al igual que las vías de infestación de los 70 niños menores de 5 años que respondieron a la definición de caso clínico.

Los síntomas más frecuentes en los casos registrados fueron los característicos de la enfermedad (mialgias, edemas generalizados y eosinofilia mayor de $4 \%$ ). No obstante, en los niños el síntoma más frecuente fue la fiebre, a diferencia de lo encontrado por otros trabajos en los que fue la diarrea (12); esta discrepancia puede deberse al bajo número de casos de triquinelosis diagnosticados en niños.
Si bien se ha informado que en Argentina la mayor frecuencia de casos se concentra en los meses de otoño e invierno (abril, mayo, junio) (13), en este estudio se encontró la mayor frecuencia en los meses de agosto, septiembre y octubre. Esta discordancia puede deberse a diferencias en las pautas culturales de consumo de carne de cerdo y sus derivados crudos o semicocidos en diferentes regiones de Argentina, aunque se requiere una evaluación más profunda en este sentido, que incluya encuestas de consumo en la población.

En Argentina se elaboran productos a base de carne de cerdos faenados y procesados en el ámbito familiar o clandestino, sin inspección veterinaria para detectar la presencia del parásito antes o después de su sacrificio (7). De hecho, se demostró que de 85 brotes de la enfermedad detectados en la provincia de Buenos Aires, 83 (97,6\%) se debieron a la ingesta de carne de cerdo cruda, seca o ahumada - como chorizos y embutidos- preparada artesanalmente (14). Los resultados encontrados en la presente investigación confirman esta tendencia, ya que según el análisis epidemiológico, la carne de cerdo fue el principal alimento involucrado en los casos clínicos y las personas que vivían en áreas rurales tenían una mayor probabilidad de enfermar.

El período de incubación descrito para la enfermedad va desde 1-43 días (3) hasta 4-20 días (5). Esa dispersión puede deberse a la forma en que transcurre la enfermedad: se extiende a 7 días en los casos clínicos graves, 16 días en infecciones moderadas, 21 días en casos benignos y 30 días en las formas abortivas (4). Sin embargo, al analizar los casos clínicos de esta investigación se encontró que, si bien la mediana se encontraba en el término de tiempo informado en la literatura científica, el período de incubación se extendía hasta 80 días en algunos casos, lo que podría deberse en parte a diferencias en la carga parasitaria ingerida por las personas afectadas. La carga infestiva con $T$. spiralis (número de larvas/g) de la carne o producto ingerido está directamente relacionada con los trastornos que ocasiona y la progresión de la enfermedad puede variar desde trastornos menores (menos de 1 larva/g) y una enfermedad leve (entre 1 y 50 larvas/g), hasta una enfermedad moderada (de 51 a 100 larvas/g), grave (de 101 a 500 larvas/g), muy grave (entre 501 y 1000 larvas/g) o crítica (más de 1000 larvas/g) (5). Se requieren estudios más específicos para poder explicar con mayor certeza este punto.

La confirmación de laboratorio en los casos que no responden totalmente a la definición clínica, constatado también en este estudio, hace reiterar la importancia de evaluar cuidadosamente la información epidemiológica y remitir al laboratorio las muestras de todos los casos sospechados.

En esta investigación no fue posible analizar los datos de los pacientes internados o la información sobre la evolución de los pacientes y sus complicaciones posteriores, ya que las fichas epidemiológicas disponibles contenían solamente la información requerida para remitir las muestras al laboratorio.

Aunque la triquinelosis es una enfermedad de información obligatoria en Argentina, los especialistas coinciden en que no todos los casos se notifican y se incorporan a los registros (7), lo que se podría subsanar si se investigaran los casos sospechados y los brotes y se generara una ficha epidemiológica integral que contuviera tanto los datos de la muestra relevantes para el laboratorio como la información necesaria para analizar los aspectos clínicos y epidemiológicos del caso. Esto implicaría, entre otras cosas, hacer una revisión de la estructura y el contenido de las fichas epidemiológicas y fortalecer el proceso de su elaboración en la práctica de los servicios de salud.

La disponibilidad de una única muestra de laboratorio positiva no debe constituir una confirmación del diagnóstico de la enfermedad, ya que se debe comprobar la seroconversión (el aumento en 
los títulos de anticuerpos) en muestras pareadas. Sin embargo, la variabilidad observada en el análisis de las muestras de cada caso por parte del laboratorio confirma la necesidad de rediscutir la norma que requiere tomar tres muestras de cada caso, en intervalos de 15 días, para confirmar la enfermedad. En esa norma, establecida por el Ministerio de Salud de la Nación en el Manual de Normas y Procedimientos de Vigilancia y Control de Enfermedades de Notificación Obligatoria, se debería considerar el empleo de más de una técnica serológica que permitan conjuntamente confirmar la infección en un mayor número de casos $(6,15)$. La técnica de ELISA presentó mayor sensibilidad y especificidad que el método de IFI cuando se emplearon ambas técnicas. Se estima que por ELISA se pueden detectar los anticuerpos generados por una larva de $T$. spiralis en 100 g de músculo estriado a partir de la segunda semana; la técnica de Western blot, por su parte, tiene aún mayor sensibilidad y especificidad que otras técnicas de laboratorio $(14,16)$.

$\mathrm{Al}$ analizar estos resultados se deben tener en cuenta algunas limitaciones. En primer lugar, este estudio se basó en el análisis de las fichas epidemiológicas remitidas por los servicios de atención sanitaria al Laboratorio Central del Ministerio de Salud de la provincia de Santa $\mathrm{Fe}$, que correspondían a muestras de casos sospechados por tratarse de pacientes con síntomas y signos compatibles con la enfermedad, pero no hay certeza de que todas las personas que padecieron la enfermedad hayan recurrido a un servicio de salud o que en todos los casos se haya considerado la triquinelosis como posible diagnóstico. En segundo lugar, no hay certeza de que los servicios de salud hayan recabado adecuadamente la información epidemiológica de los casos sospechados y la hayan incorporado adecuadamente a la ficha. Se debe, además, tener en cuenta que el diagnóstico confirmatorio pudo estar incompleto por la falta de muestras seriadas o fallas en el procedimiento. Por último, para este estudio, solo se tuvo información de los servicios públicos de salud. Todas estas limitaciones, no obstante, podrían sesgar la información en el sentido de un subregistro.

A pesar de estas limitaciones, los resultados muestran que a partir del año 2001, el número de casos clínicos informados disminuyó paulatinamente, con un ligero aumento en el último bienio. El mayor número de brotes y casos de triquinelosis se registró en los de- partamentos del centro y el sur de la provincia, donde se concentran la producción porcina y la mayor población. Esta situación alerta sobre la necesidad de diseñar y poner en práctica acciones específicas de prevención y control de la enfermedad en estos departamentos.

Se confirmó la utilidad del análisis epidemiológico de riesgos como herramienta complementaria para evaluar, gestionar y comunicar los riesgos sanitarios de triquinelosis. Se deben modificar las fichas epidemiológicas y realizar actividades específicas de entrenamiento en los servicios de salud para su correcta confección e incorporar en ellas los datos necesarios para el análisis de las situaciones epidemiológicas que se presentan. Se recomienda incluir las pruebas de ELISA o Western blot, además de las pruebas por IFI, en el esquema de diagnóstico y confirmación de laboratorio de los casos sospechados de triquinelosis. Se deben reforzar las medidas dirigidas a erradicar los criaderos de cerdos sin control sanitario y reforzar las acciones de sensibilización de la población para desestimular el consumo de chacinados que no tengan la certificación sanitaria oficial.

\section{Conflictos de intereses. Ninguno.}

\section{REFERENCIAS}

1. Krivokapich S, Molina V, Bergagna $H$, Guarnera E. Epidemiological survey of trichinella infection in domestic, synanthropic, and sylvatic animals from Argentina. J Helminthol. 2006;80:267-9.

2. Sequeira G. Aspectos epidemiológicos de la triquinelosis en la ciudad de Santa Fe, República Argentina: los roedores en el ciclo biológico de la enfermedad [tesis de maestría]. Santa Fe, Argentina: Facultad de Ciencias Veterinarias, Universidad Nacional del Litoral; 2001.

3. Acha PN, Szyfres B. Triquinelosis. En: Organización Panamericana de la Salud. Zoonosis y enfermedades transmisibles comunes al hombre y a los animales. Vol. III. Parasitosis. 3. a ed. Washington, D.C.: OPS; 2003. Pp. 325-39.

4. Koci cka W. Trichinellosis: human disease, diagnosis, and treatment. Vet Parasitol. 2000;93(3-4):365-83.

5. Ramírez Valenzuela M. Epidemiología de la triquinelosis. En: Moreno Chan R. Ciencia veterinaria. Vol. III. México D.F.: Universidad Nacional Autónoma de México; 1981.
Pp. 278-325. Disponible en: http://www. fmvz.unam. $\mathrm{mx} / \mathrm{fmvz} /$ cienciavet/indices/ CvVol003.htm Acceso el 10 de abril de 2013.

6. Ambrosioni J, Cecchini D, Castellaro P. Triquinosis: aspectos epidemiológicos, clínicos y de laboratorio. Estudio retrospectivo a 10 años (1994-2003). Enferm Infecc Microbiol Clin. 2006;24(7):440-4.

7. Caracostantogolo J, Steffan P, Dillon J, De la Sota M, Belgrano D, Veneroni $R$, et al. Mejoramiento del control de la triquinelosis en Argentina: Proyecto TCP ARG 3003. En: Organización de las Naciones Unidas para la Agricultura y la Alimentación. Mejoramiento del control de la triquinelosis. Roma: FAO; 2007. Pp. 3-66.

8. Ribicich M, Gamble HR, Bolpe J, Sommerfelt I, Cardillo N, Scialfa E, et al. Evaluation of the risk of transmission of Trichinella in pork production systems in Argentina. Vet Parasitol. 2009;159:350-3.

9. Organización de las Naciones Unidas para la Agricultura y la Alimentación, Oficina Regional de la FAO para América Latina y el Caribe. Análisis de riesgos relativos a la inocuidad de los alimentos. Guía para las autoridades nacionales de inocuidad de los alimentos. Roma: FAO; 2007. (Estudio FAO, Alimentación y Nutrición 87). Disponible en: http://www.rlc.fao.org/es/temas/inocui dad/analisis-de-riesgos/ Acceso el 3 de abril de 2013.

10. Zelko CN, Vicario TV. Análisis de la cadena de valor de la producción de carne porcina en la provincia de Santa Fe. Santa Fe, Argentina: Secretaría de Sistemas Agropecuarios, Agroalimentos y Biocombustibles, Ministerio de la Producción de la Provincia de Santa Fe; 2006.

11. Argentina, Ministerio de Salud de la Nación. Boletín Epidemiológico Anual. Buenos Aires: Ministerio de Salud de la Nación, Dirección de Epidemiología; 2007. Disponible en: http:// msal.gov.ar/htm/site/sala_situacion/ PANELES/boletines / BEPANUAL_2007. parte1.pdf Acceso el 10 de mayo de 2013.

12. Calcagno MA, Teixeira $C$, Forastiero MA Costantino SN, Venturiello SM. Aspectos clínicos, serológicos y parasitológicos de un brote de triquinelosis humana en Villa Mercedes, San Luís, Argentina. Las fases aguda 
y crónica de la infección. Medicina (Buenos Aires). 2005;65(4):302-6.

13. Ribicich M, Gamble HR, Rosa A, Bolpe J, Franco A. Trichinellosis in Argentina: an historical review. Vet Parasitol. 2005;132:137-42.

14. Guanera E, Krivokapich S, Peralta J, Trabattoni E, Paoletti C, Baravalle A, et al. Sistema de diagnóstico inmunológico de triquinelosis porcina ante-mortem. Sitio Argentino de Producción Animal [sitio en Internet]; 2006. Dis- ponible en: http://www.produccion-animal. com.ar Acceso el 3 de abril de 2013.

15. Barlasina MS, Pedevilla C, Kade P, Costantino SN, Taus MR, Venturiello SM. Trichinellosis serology in blood-donors from a non-endemic area of Argentina. Medicina (Buenos Aires). 2009;69(3):297-301.

16. Gajadhar AA, Pozio E, Ray Gamble $\mathrm{H}$, Nöckler K, Maddox-Hyttel C, Forbes LB, et al. Trichinella diagnostics and control: mandatory and best practices for ensuring food safety. Vet Parasitol. 2009;159(3-4): 197-205.

Manuscrito recibido el 16 de mayo de 2012. Aceptado para publicación, tras revisión, el 28 de diciembre de 2012.

ABSTRACT Objective. To characterize recorded trichinosis cases and outbreaks in Santa Fe Province, Argentina, from 1998 to 2009 from epidemiological, clinical, and laboratory viewpoints.

Epidemiological study of recorded trichinosis cases in Santa Fe Province, Argentina,

Methods. An analysis was conducted of 1519 epidemiological records of persons who had signs and symptoms compatible with trichinosis in Santa Fe Province in the period 1998-2009. A descriptive statistical analysis of information in epidemiological records and of clinical and laboratory results, as well as a bifactorial analysis of possible associations with epidemiological and environmental factors were also carried out.

Results. Trichinosis symptoms were found in 1276 cases; 372 met the clinical case definition and 224 samples were positive by indirect immunofluorescence, independent of their clinical case classification. There were 27 outbreaks that involved 1157 cases identified in eight departments, with greater frequency in the province's center and south. Annual distribution of epidemiologically associated cases was heterogeneous, with greater frequency in the period 2000-2003 and in the months of August to October. Probability of becoming sick was greater with consumption of products from informal sources $(\mathrm{OR}=3.69 ; P=0.014)$ and in rural areas $(\mathrm{OR}=1.799$; $P=0.011$ ). Disease incubation period (median) was 12 days. The risk of becoming sick was 2.06 times greater in persons who ate meat or ate pork by-products than in those who did not.

Conclusions. The greatest number of outbreaks occurred in departments that were more populous and where pork production was greater. Specific actions should be designed for prevention and control of the disease in those departments. Epidemiological risk analysis should be considered in evaluating, managing, and communicating trichinosis health risks.

Key words Trichinellosis; swine; epidemiology; Argentina. 